\title{
Examples of smooth compact toric varieties that are not quasitoric manifolds
}

\author{
YUSUKE SUYAMA
}

\begin{abstract}
We construct smooth compact toric varieties of complex dimension $\geq 4$ whose orbit spaces by the action of the compact torus are not homeomorphic to simple polytopes (as manifolds with corners). These provide the first known examples of smooth compact toric varieties that are not quasitoric manifolds.
\end{abstract}

52B05; 14M25, 57S15

\section{Introduction}

A toric variety $X$ of complex dimension $n$ is a normal algebraic variety over $\mathbb{C}$ containing the algebraic torus $\left(\mathbb{C}^{*}\right)^{n}$ as an open dense subset, such that the natural action of $\left(\mathbb{C}^{*}\right)^{n}$ on itself extends to $X$, where $\mathbb{C}^{*}=\mathbb{C} \backslash\{0\}$. In this paper, a smooth compact toric variety is called a toric manifold. The orbit space $X /\left(S^{1}\right)^{n}$ of a toric manifold $X$ by the restricted action of the compact torus $\left(S^{1}\right)^{n} \subset\left(\mathbb{C}^{*}\right)^{n}$ is a manifold with corners such that all faces are contractible and any non-empty intersection of faces is connected. If $X$ is projective, then a moment map identifies the orbit space $X /\left(S^{1}\right)^{n}$ with a simple polytope, so the orbit space is homeomorphic to a simple polytope as a manifold with corners. Any toric manifold of complex dimension $\leq 2$ is projective. Although there are many non-projective toric manifolds in complex dimension 3, their orbit spaces are all homeomorphic to simple polytopes as manifolds with corners; this follows from Steinitz's theorem on planar graphs. It has so far been unknown whether this is still the case in higher dimensions.

The purpose of this paper is to prove the following theorem:

Theorem 1 For any integer $n \geq 4$, there are infinitely many smooth compact toric varieties $X$ of complex dimension $n$ whose orbit spaces $X /\left(S^{1}\right)^{n}$ are not homeomorphic to any simple polytope as manifolds with corners.

A quasitoric manifold $X$ of (real) dimension $2 n$ over a simple polytope $P$ is a closed smooth manifold with a smooth action of $\left(S^{1}\right)^{n}$ such that the action is locally standard and the orbit space $X /\left(S^{1}\right)^{n}$ is the simple polytope $P$ [4]. The restricted action 
of $\left(S^{1}\right)^{n}$ on a toric manifold of complex dimension $n$ is always locally standard. Therefore, if a toric manifold is projective or of dimension $n \leq 3$, then it is a quasitoric manifold [2]. Our theorem implies that if $n \geq 4$, then there are infinitely many toric manifolds of complex dimension $n$ that are not quasitoric manifolds. This solves a problem posed by Buchstaber and Panov [2, Problem 5.20]. In a 2003 preprint, Y Civan [3] claimed the existence of a toric manifold as in Theorem 1, but it has been recognized that his explanation of non-polytopality was insufficient. Our construction is based on ideas found in [3] but is more explicit.

A simplicial $n-$ sphere is a simplicial complex that is homeomorphic to $S^{n}$. A simplicial sphere is polytopal if it is combinatorially equivalent to the boundary complex of a simplicial polytope. The orbit space $X /\left(S^{1}\right)^{n}$ of a toric manifold $X$ is homeomorphic to a simple convex polytope if and only if the underlying simplicial complex of the fan of $X$ is polytopal [2]. The Barnette sphere is a simplicial 3-sphere which is not polytopal [1]. However, the Barnette sphere cannot be the underlying simplicial complex of a non-singular fan [6, Theorem 9.1]. So we first find a simplicial singular fan whose underlying simplicial complex is the Barnette sphere and change it into a non-singular fan by subdivision while keeping the non-polytopality of the underlying simplicial complex. Our proof of non-polytopality is similar to that of [5, Theorem 5.3, Chapter III]. Thus we obtain a desired toric manifold $X$ of complex dimension 4. In fact, we can produce infinitely many such toric manifolds by performing subdivisions and suspensions on the fan of $X$.

The structure of the paper is as follows: In Section 2, we give a fan whose underlying simplicial complex is the Barnette sphere. In Section 3, we prove Theorem 1.

\section{The Barnette sphere}

The Barnette sphere is a simplicial 3-sphere consisting of the 8 vertices $e_{1}, e_{2}, e_{3}$, $e_{4}, d_{1}, d_{2}, d_{3}, d_{4}$ described in Figure 1; see [1] for details.

We assign to the vertices of the Barnette sphere certain points in $\mathbb{R}^{4}$ as follows: $e_{1}, e_{2}, e_{3}, e_{4}$ are the standard basis of $\mathbb{Z}^{4} \subset \mathbb{R}^{4}$ and

$$
\begin{array}{ll}
d_{1}=(-1,0,-2,1), & d_{2}=(-2,-1,0,1), \\
d_{3}=(0,-2,-1,1), & d_{4}=(1,0,1,-1) .
\end{array}
$$

Let $\Delta$ be the set consisting of 194 -dimensional cones as in Table 1 and their faces. The determinants of the matrices formed by the four edge vectors of each 4-dimensional cone in $\Delta$ are also noted in Table 1. 


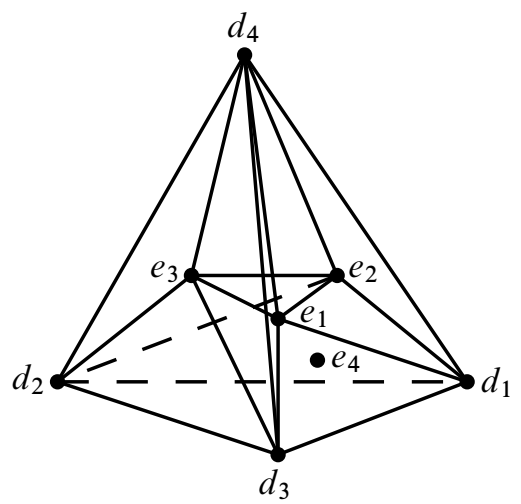

Figure 1: Diagram of the Barnette sphere (some edges are omitted)

\begin{tabular}{lcr|ccc|ccr} 
cone & edges & det & cone & edges & det & cone & edges & det \\
\hline$\sigma_{1}$ & $e_{1} e_{2} e_{3} e_{4}$ & 1 & $\sigma_{8}$ & $d_{1} e_{2} d_{3} e_{4}$ & 1 & $\sigma_{15}$ & $e_{1} d_{1} d_{3} d_{4}$ & 2 \\
$\sigma_{2}$ & $d_{1} e_{2} e_{3} e_{4}$ & -1 & $\sigma_{9}$ & $e_{1} d_{2} e_{3} d_{3}$ & 1 & $\sigma_{16}$ & $d_{1} e_{2} d_{2} d_{4}$ & 1 \\
$\sigma_{3}$ & $e_{1} d_{2} e_{3} e_{4}$ & -1 & $\sigma_{10}$ & $e_{1} e_{2} d_{3} d_{1}$ & 1 & $\sigma_{17}$ & $d_{3} d_{2} e_{3} d_{4}$ & 3 \\
$\sigma_{4}$ & $e_{1} e_{2} d_{3} e_{4}$ & -1 & $\sigma_{11}$ & $d_{1} e_{2} e_{3} d_{2}$ & 1 & $\sigma_{18}$ & $d_{1} d_{2} d_{3} e_{4}$ & -9 \\
$\sigma_{5}$ & $e_{1} e_{2} e_{3} d_{4}$ & -1 & $\sigma_{12}$ & $e_{1} e_{2} d_{1} d_{4}$ & 1 & $\sigma_{19}$ & $d_{1} d_{2} d_{3} d_{4}$ & 3 \\
$\sigma_{6}$ & $d_{1} d_{2} e_{3} e_{4}$ & 1 & $\sigma_{13}$ & $e_{1} d_{3} e_{3} d_{4}$ & 2 & & & \\
$\sigma_{7}$ & $e_{1} d_{2} d_{3} e_{4}$ & 1 & $\sigma_{14}$ & $d_{2} e_{2} e_{3} d_{4}$ & 1 & & &
\end{tabular}

Table 1: 4-dimensional cones in $\Delta$

Lemma $2 \Delta$ is a simplicial complete fan.

Proof By construction, any $k$-dimensional cone of $\Delta$ is generated by linearly independent $k$ vectors in $\mathbb{Z}^{4}$. So any cone in $\Delta$ is simplicial. One can check that for each 3dimensional cone $\tau$ in $\Delta$, the two 4-dimensional cones containing it as a common face have no intersection except $\tau$. For example, let $\tau$ be the 3 -dimensional cone generated by $d_{1}, e_{2}, d_{3}$. The cones containing $\tau$ are $\sigma_{8}$ and $\sigma_{10}$. Since $e_{1}+e_{4}=-d_{1}+4 e_{2}+2 d_{3}$, $\sigma_{8}$ and $\sigma_{10}$ are on opposite sides of the 3 -subspace containing $\tau$. This implies that $\mathbb{R}^{4}$ is covered by the 4 -dimensional cones uniformly. Hence if some cones overlap, then every cone is covered by the union of some other cones. However, one can check by elementary calculations that, for example, each of $\sigma_{2}, \ldots, \sigma_{19}$ has no points whose coordinates are all positive (that is, interior points of $\sigma_{1}$ ). For example, if $a_{1} e_{1}+a_{2} e_{2}+a_{3} d_{1}+a_{4} d_{4} \in \sigma_{12}\left(a_{i} \geq 0\right)$ is an interior point of $\sigma_{1}$, then we must have $a_{1}-a_{3}+a_{4}>0, a_{2}>0,-2 a_{3}+a_{4}>0, a_{3}-a_{4}>0$. The latter two inequalities imply $-a_{3}>0$, which is a contradiction. So there are no overlaps among the 4-dimensional cones in $\Delta$, which means that $\Delta$ is a complete fan. This completes the proof. 
Remark A computer calculation shows that the $81^{4}=43,046,721$ lattice points in

$$
\left\{\left(x_{1}, x_{2}, x_{3}, x_{4}\right) \in \mathbb{Z}^{4} \mid x_{i} \in \mathbb{Z},-40 \leq x_{i} \leq 40\right\}
$$

are classified into the five types in Table 2 . The 260 relative interior points of the common 1-face of more than two cones are

$\left\{m e_{1}, m e_{2}, m e_{3}, m e_{4}, n d_{1}, n d_{2}, n d_{3}, m d_{4} \in \mathbb{Z}^{4} \mid m, n \in \mathbb{Z}, 1 \leq m \leq 40,1 \leq n \leq 20\right\}$.

The relative interior point of the common 0 -face of more than two cones is the origin. The sum of the numbers in Table 2 is $81^{4}$, which supports the completeness of the fan $\Delta$.

\begin{tabular}{l|r} 
Classification & \# \\
\hline Interior points of a cone & $41,315,292$ \\
Rel. int. points of the common facet of two cones & $1,696,978$ \\
Rel. int. points of the common 2-face of more than two cones & 34,190 \\
Rel. int. points of the common 1-face of more than two cones & 260 \\
Rel. int. point of the common 0-face of more than two cones & 1
\end{tabular}

Table 2: Classification of lattice points

\section{Proof of Theorem 1}

According to Table 1, the singular 4-dimensional cones of $\Delta$ are $\sigma_{13}, \sigma_{15}, \sigma_{17}, \sigma_{18}$ and $\sigma_{19}$. We shall subdivide them so that the resulting 4 -dimensional cones are all non-singular. We denote a cone by arranged edge vectors in $\mathbb{R}^{4}$ (eg $\left.\sigma_{13}=e_{1} d_{3} e_{3} d_{4}\right)$.

Subdivision of $\sigma_{13}$ and $\sigma_{15}$ We introduce a point

$$
c_{1}=\frac{1}{2} e_{1}+\frac{1}{2} d_{3}+\frac{1}{2} d_{4}=(1,-1,0,0) .
$$

Note that $c_{1}$ is on the 3 -dimensional cone $e_{1} d_{3} d_{4}$. We subdivide the cones $\sigma_{13}$ and $\sigma_{15}$ as (see Figure 2)

$$
\begin{array}{lllll}
\sigma_{13}=e_{1} d_{3} e_{3} d_{4} & \text { into } c_{1} d_{3} e_{3} d_{4}, & e_{1} c_{1} e_{3} d_{4}, & e_{1} d_{3} e_{3} c_{1}, \\
\sigma_{15}=e_{1} d_{1} d_{3} d_{4} & \text { into } c_{1} d_{1} d_{3} d_{4}, & e_{1} d_{1} c_{1} d_{4}, & e_{1} d_{1} d_{3} c_{1} .
\end{array}
$$

All the determinants of the resulting cones are 1 .

Subdivision of $\sigma_{17}$ We introduce a point

$$
c_{2}=\frac{1}{3} d_{3}+\frac{1}{3} d_{2}+\frac{2}{3} e_{3}+\frac{2}{3} d_{4}=(0,-1,1,0)
$$




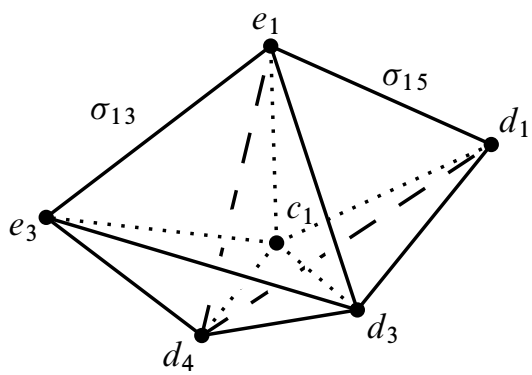

Figure 2: Subdivision of $\sigma_{13}$ and $\sigma_{15}$

and subdivide $\sigma_{17}=d_{3} d_{2} e_{3} d_{4}$ into

$$
c_{2} d_{2} e_{3} d_{4}, \quad d_{3} c_{2} e_{3} d_{4}, d_{3} d_{2} c_{2} d_{4}, d_{3} d_{2} e_{3} c_{2} .
$$

The determinants of the cones $d_{3} d_{2} c_{2} d_{4}, d_{3} d_{2} e_{3} c_{2}$ are 2 . So we further introduce a point

$$
c_{3}=\frac{1}{2} d_{3}+\frac{1}{2} d_{2}+\frac{1}{2} c_{2}=(-1,-2,0,1) .
$$

Note that $c_{3}$ is on the 3-dimensional cone $d_{3} d_{2} c_{2}$. We subdivide the cones $d_{3} d_{2} c_{2} d_{4}$, $d_{3} d_{2} e_{3} c_{2}$ as (see Figure 3)

$$
\begin{array}{lllll}
d_{3} d_{2} c_{2} d_{4} & \text { into } c_{3} d_{2} c_{2} d_{4}, & d_{3} c_{3} c_{2} d_{4}, & d_{3} d_{2} c_{3} d_{4}, \\
d_{3} d_{2} e_{3} c_{2} & \text { into } c_{3} d_{2} e_{3} c_{2}, & d_{3} c_{3} e_{3} c_{2}, & d_{3} d_{2} e_{3} c_{3} .
\end{array}
$$

All the determinants of the resulting cones are 1 .

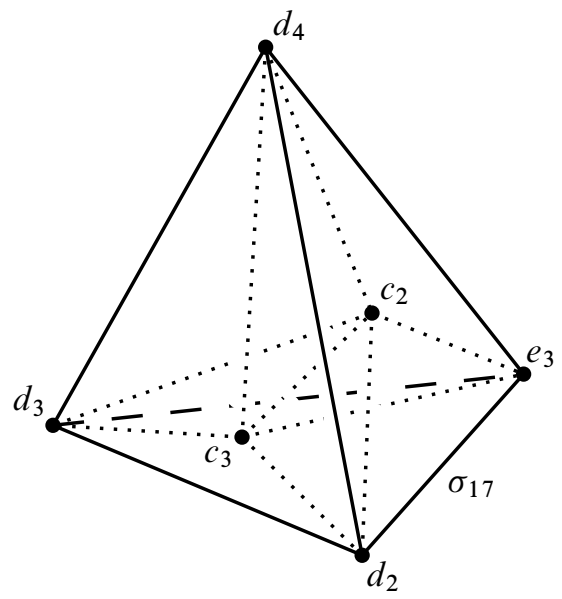

Figure 3: Subdivision of $\sigma_{17}$

Subdivision of $\sigma_{18}$ We introduce a point

$$
c_{4}=\frac{1}{3} d_{1}+\frac{1}{3} d_{2}+\frac{1}{3} d_{3}=(-1,-1,-1,1) .
$$


Note that $c_{4}$ is on the 3 -dimensional cone $d_{1} d_{2} d_{3}$. We subdivide $\sigma_{18}=d_{1} d_{2} e_{3} d_{4}$ into $c_{4} d_{2} d_{3} e_{4}, d_{1} c_{4} d_{3} e_{4}, d_{1} d_{2} c_{4} e_{4}$. All the determinants of the resulting cones are -3 . So we further introduce points

$$
\begin{aligned}
& c_{5}=\frac{1}{3} c_{4}+\frac{1}{3} d_{2}+\frac{2}{3} d_{3}+\frac{2}{3} e_{4}=(-1,-2,-1,2), \\
& c_{7}=\frac{2}{3} d_{1}+\frac{1}{3} c_{4}+\frac{1}{3} d_{3}+\frac{2}{3} e_{4}=(-1,-1,-2,2), \\
& c_{9}=\frac{1}{3} d_{1}+\frac{2}{3} d_{2}+\frac{1}{3} c_{4}+\frac{2}{3} e_{4}=(-2,-1,-1,2)
\end{aligned}
$$

and we subdivide the cones $c_{4} d_{2} d_{3} e_{4}, d_{1} c_{4} d_{3} e_{4}, d_{1} d_{2} c_{4} e_{4}$ as

$$
\begin{array}{llllll}
c_{4} d_{2} d_{3} e_{4} & \text { into } & c_{5} d_{2} d_{3} e_{4}, & c_{4} c_{5} d_{3} e_{4}, & c_{4} d_{2} c_{5} e_{4}, & c_{4} d_{2} d_{3} c_{5}, \\
d_{1} c_{4} d_{3} e_{4} & \text { into } & c_{7} c_{4} d_{3} e_{4}, & d_{1} c_{7} d_{3} e_{4}, & d_{1} c_{4} c_{7} e_{4}, & d_{1} c_{4} d_{3} c_{7}, \\
d_{1} d_{2} c_{4} e_{4} & \text { into } & c_{9} d_{2} c_{4} e_{4}, & d_{1} c_{9} c_{4} e_{4}, & d_{1} d_{2} c_{9} e_{4}, & d_{1} d_{2} c_{4} c_{9} .
\end{array}
$$

The determinants of the cones $c_{4} d_{2} c_{5} e_{4}, c_{4} d_{2} d_{3} c_{5}, c_{7} c_{4} d_{3} e_{4}, d_{1} c_{4} d_{3} c_{7}, d_{1} c_{9} c_{4} e_{4}$ and $d_{1} d_{2} c_{4} c_{9}$ are -2 . So we further introduce points

$$
\begin{aligned}
& c_{6}=\frac{1}{2} c_{4}+\frac{1}{2} d_{2}+\frac{1}{2} c_{5}=(-2,-2,-1,2), \\
& c_{8}=\frac{1}{2} c_{7}+\frac{1}{2} c_{4}+\frac{1}{2} d_{3}=(-1,-2,-2,2), \\
& c_{10}=\frac{1}{2} d_{1}+\frac{1}{2} c_{9}+\frac{1}{2} c_{4}=(-2,-1,-2,2)
\end{aligned}
$$

and we subdivide the cones as (see Figure 4)

$$
\begin{array}{lllll}
c_{4} d_{2} c_{5} e_{4} & \text { into } c_{6} d_{2} c_{5} e_{4}, & c_{4} c_{6} c_{5} e_{4}, & c_{4} d_{2} c_{6} e_{4}, \\
c_{4} d_{2} d_{3} c_{5} & \text { into } c_{6} d_{2} d_{3} c_{5}, & c_{4} c_{6} d_{3} c_{5}, & c_{4} d_{2} d_{3} c_{6}, \\
c_{7} c_{4} d_{3} e_{4} & \text { into } c_{8} c_{4} d_{3} e_{4}, & c_{7} c_{8} d_{3} e_{4}, & c_{7} c_{4} c_{8} e_{4}, \\
d_{1} c_{4} d_{3} c_{7} & \text { into } d_{1} c_{8} d_{3} c_{7}, & d_{1} c_{4} c_{8} c_{7}, & d_{1} c_{4} d_{3} c_{8}, \\
d_{1} c_{9} c_{4} e_{4} & \text { into } c_{10} c_{9} c_{4} e_{4}, & d_{1} c_{10} c_{4} e_{4}, & d_{1} c_{9} c_{10} e_{4}, \\
d_{1} d_{2} c_{4} c_{9} & \text { into } & c_{10} d_{2} c_{4} c_{9}, & d_{1} d_{2} c_{10} c_{9}, & d_{1} d_{2} c_{4} c_{10} .
\end{array}
$$

All the determinants of the resulting cones are 1 .

Subdivision of $\sigma_{19}$ We subdivide $\sigma_{19}=d_{1} d_{2} d_{3} d_{4}$ into $c_{4} d_{2} d_{3} d_{4}, d_{1} c_{4} d_{3} d_{4}$, $d_{1} d_{2} c_{4} d_{4}$. All the determinants of the resulting cones are 1 .

Thus we replaced $\sigma_{13}, \sigma_{15}, \sigma_{17}, \sigma_{18}$ and $\sigma_{19}$ by the cones in Table 3 .

Now we have a refinement $\Delta^{\prime}$ of $\Delta$ which has 18 edges and 554 -dimensional cones. The determinant of each 4-dimensional cone of $\Delta^{\prime}$ is 1 or -1 . So $\Delta^{\prime}$ is a non-singular complete fan and the corresponding toric variety $X\left(\Delta^{\prime}\right)$ is a toric manifold. 


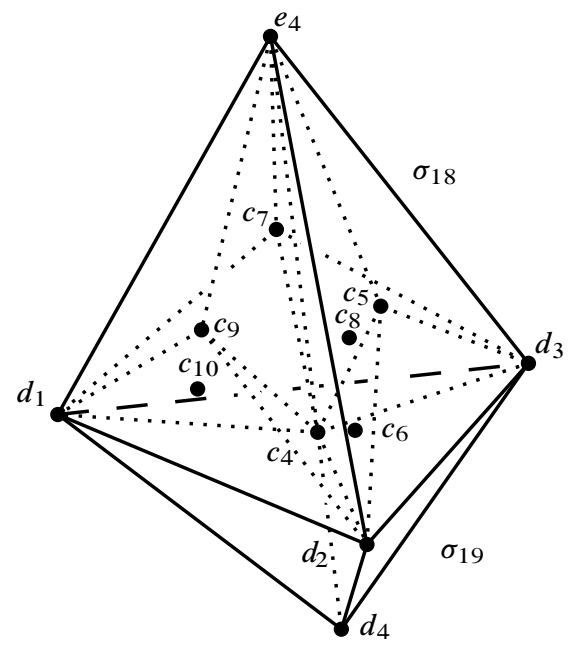

Figure 4: Subdivision of $\sigma_{18}$ and $\sigma_{19}$

\begin{tabular}{cc|cc|cc|cc} 
cone & sign & cone & sign & cone & sign & cone & sign \\
\hline$c_{1} d_{3} e_{3} d_{4}$ & + & $c_{3} d_{2} e_{3} c_{2}$ & + & $c_{8} c_{4} d_{3} e_{4}$ & - & $d_{1} c_{9} c_{10} e_{4}$ & - \\
$e_{1} c_{1} e_{3} d_{4}$ & + & $d_{3} c_{3} e_{3} c_{2}$ & + & $c_{7} c_{8} d_{3} e_{4}$ & - & $d_{1} d_{2} c_{9} e_{4}$ & - \\
$e_{1} d_{3} e_{3} c_{1}$ & + & $d_{3} d_{2} e_{3} c_{3}$ & + & $c_{7} c_{4} c_{8} e_{4}$ & - & $c_{10} d_{2} c_{4} c_{9}$ & - \\
$c_{1} d_{1} d_{3} d_{4}$ & + & $c_{5} d_{2} d_{3} e_{4}$ & - & $d_{1} c_{7} d_{3} e_{4}$ & - & $d_{1} d_{2} c_{10} c_{9}$ & - \\
$e_{1} d_{1} c_{1} d_{4}$ & + & $c_{4} c_{5} d_{3} e_{4}$ & - & $d_{1} c_{4} c_{7} e_{4}$ & - & $d_{1} d_{2} c_{4} c_{10}$ & - \\
$e_{1} d_{1} d_{3} c_{1}$ & + & $c_{6} d_{2} c_{5} e_{4}$ & - & $d_{1} c_{8} d_{3} c_{7}$ & - & $c_{4} d_{2} d_{3} d_{4}$ & + \\
$c_{2} d_{2} e_{3} d_{4}$ & + & $c_{4} c_{6} c_{5} e_{4}$ & - & $d_{1} c_{4} c_{8} c_{7}$ & - & $d_{1} c_{4} d_{3} d_{4}$ & + \\
$d_{3} c_{2} e_{3} d_{4}$ & + & $c_{4} d_{2} c_{6} e_{4}$ & - & $d_{1} c_{4} d_{3} c_{8}$ & - & $d_{1} d_{2} c_{4} d_{4}$ & + \\
$c_{3} d_{2} c_{2} d_{4}$ & + & $c_{6} d_{2} d_{3} c_{5}$ & - & $c_{9} d_{2} c_{4} e_{4}$ & - & & \\
$d_{3} c_{3} c_{2} d_{4}$ & + & $c_{4} c_{6} d_{3} c_{5}$ & - & $c_{10} c_{9} c_{4} e_{4}$ & - & & \\
$d_{3} d_{2} c_{3} d_{4}$ & + & $c_{4} d_{2} d_{3} c_{6}$ & - & $d_{1} c_{10} c_{4} e_{4}$ & - & &
\end{tabular}

Table 3: Subdivided cones

Proposition 3 The underlying simplicial complex $K_{\Delta^{\prime}}$ is not polytopal. So the orbit space $X\left(\Delta^{\prime}\right) /\left(S^{1}\right)^{n}$ of the corresponding toric manifold $X\left(\Delta^{\prime}\right)$ is not homeomorphic to any simple polytope as a manifold with corners, that is, $X\left(\Delta^{\prime}\right)$ is not a quasitoric manifold.

Proof Our proof is similar to the proof of [5, 5.3 Theorem, Chapter III]. Suppose that $K_{\Delta^{\prime}}$ is polytopal. We denote the 3 -simplex corresponding to $\sigma_{i}$ by $A_{i}$ and denote a 3 -simplex by its arranged vertices. Take a Schlegel diagram of $K_{\Delta^{\prime}}$ to the 
3-simplex $A_{11}=d_{1} e_{2} e_{3} d_{2}$. The two 3-simplices $A_{2}$ and $A_{6}$ intersect along the common face $d_{1} e_{3} e_{4}$, and the point $c_{1}$ is not in $A_{2} \cup A_{6}$. The star st $\left(e_{1} d_{3}\right)$ of the edge $e_{1} d_{3}$ is the union of the six 3-simplices $A_{9}=e_{1} d_{2} e_{3} d_{3}, e_{1} d_{3} e_{3} c_{1}, e_{1} d_{1} d_{3} c_{1}$, $A_{10}=e_{1} e_{2} d_{3} d_{1}, A_{4}=e_{1} e_{2} d_{3} e_{4}, A_{7}=e_{1} d_{2} d_{3} e_{4}$. Since the link $\operatorname{lk}\left(e_{1} d_{3}\right)$ of $e_{1} d_{3}$ consists of the simplices in st $\left(e_{1} d_{3}\right)$ which do not intersect $e_{1} d_{3}$, it consists of six edges $d_{2} e_{3}, e_{3} c_{1}, c_{1} d_{1}, d_{1} e_{2}, e_{2} e_{4}, e_{4} d_{2}$. In the Schlegel diagram, $c_{1}$ is in the interior of $A_{11}$ and is in the exterior of $A_{2}=d_{1} e_{2} e_{3} e_{4}$ and $A_{6}=d_{1} d_{2} e_{3} e_{4}$ since $c_{1}$ is not in $A_{2}$ nor in $A_{6}$. Hence either $e_{3} c_{1}$ or $c_{1} d_{1}$ passes through the triangle $e_{2} e_{4} d_{2}$. So the two triangles $e_{2} e_{4} d_{2}$ and $e_{3} c_{1} d_{1}$ are linked as links of a chain (see Figure 5).

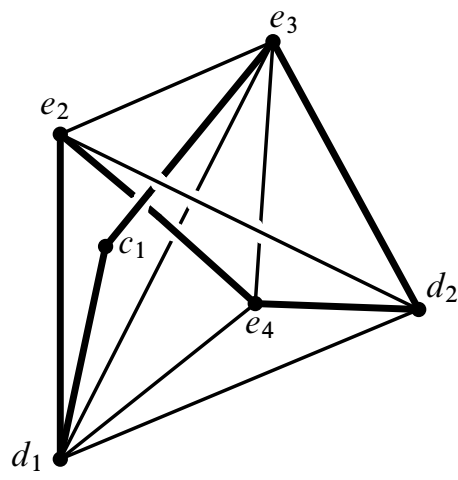

Figure 5: Schlegel diagram of $K_{\Delta^{\prime}}$ to the simplex $A_{11}$
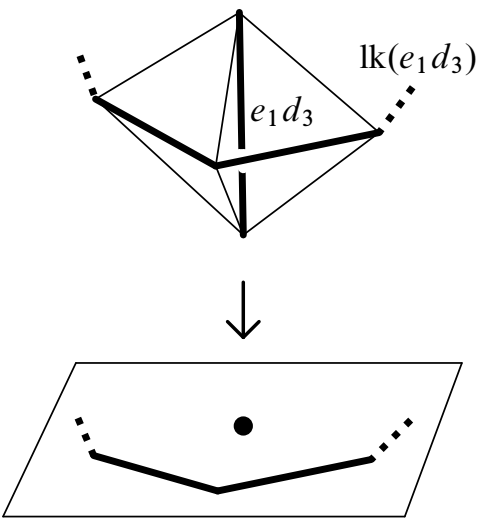

Figure 6: Edge, link and their projections

Consider the projection of the diagram Figure 5 onto a plane. Since $e_{2} e_{4} d_{2}$ and $e_{3} c_{1} d_{1}$ are linked, their images on the plane intersect. Thus the image of the diagram in Figure 5 onto a plane falls into seven types in Figure 7 essentially. The former three diagrams in Figure 7 are the case where each point of $d_{1}, e_{2}, e_{3}, d_{2}$ is a boundary point of the image of $A_{11}$, and the latter four diagrams in Figure 7 are the case where one point of $d_{1}, e_{2}, e_{3}, d_{2}$ is an interior point of the image of $A_{11}$. The positions of the points $e_{4}$ and $c_{1}$ may differ from the graphs, but in any case, the image of $\operatorname{lk}\left(e_{1} d_{3}\right)$ has a self-intersection.

However, if the simplicial complex is polytopal, the link of any edge can be projected onto a plane perpendicular to the affine hull of the edge without self-intersection [5, Theorem 5.3, Chapter III]. Indeed, the 3 -simplices of $\operatorname{st}\left(e_{1} d_{3}\right)$ are attached to $e_{1} d_{3}$ along their faces and $\operatorname{lk}\left(e_{1} d_{3}\right)$ is projected without self-intersection (see Figure 6). This is a contradiction. Thus we proved the proposition.

The main theorem is deduced by using the fan $\Delta^{\prime}$. 


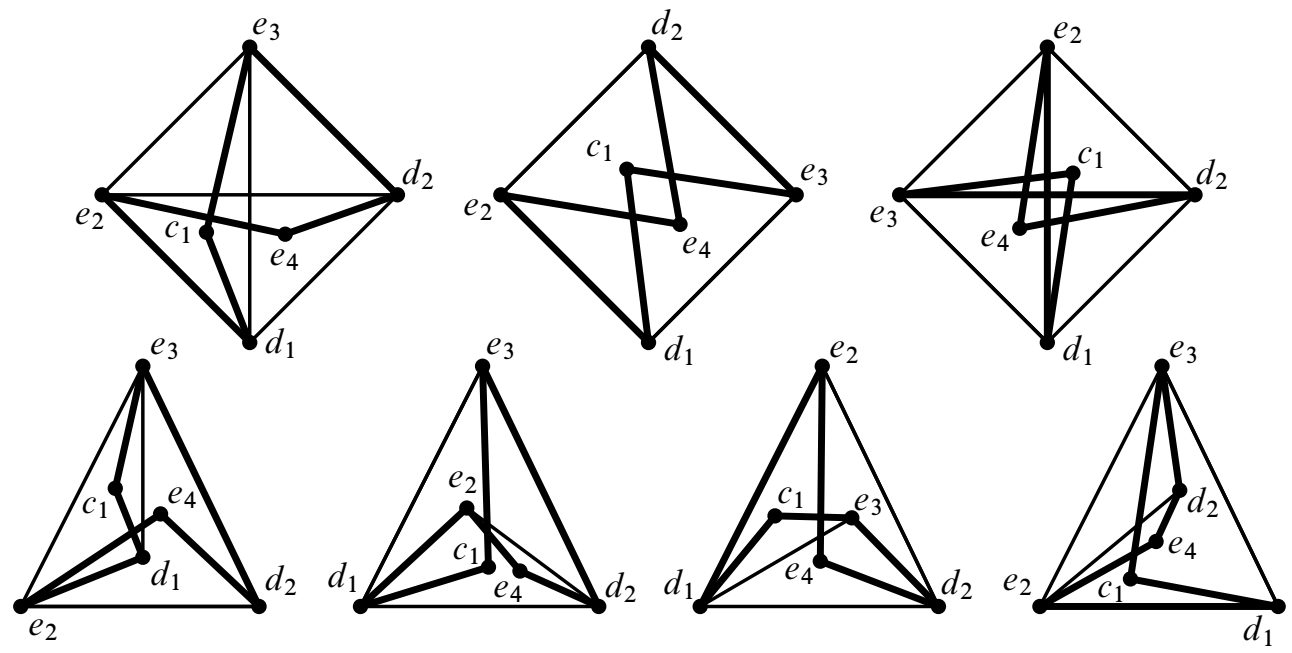

Figure 7: Images of the Schlegel diagram (up to mirror images)

Proof of Theorem 1 Subdividing the cone $\sigma_{16}$ in $\Delta^{\prime}$ by its interior point $d_{1}+e_{2}+$ $d_{2}+d_{4}$, we have another toric manifold of complex dimension 4 whose orbit space by the compact torus is not homeomorphic to any simple polytope as a manifold with corners. Successive subdivisions produce infinitely many such toric manifolds.

If a simplicial sphere is non-polytopal, then its suspension is also non-polytopal. Because if its suspension were polytopal, the link of a new vertex would also be polytopal, which contradicts that the link is the original non-polytopal simplicial sphere. Thus for any $n \geq 4$, we have infinitely many toric manifolds of complex dimension $n$ whose orbit spaces by the compact torus are not homeomorphic to any simple polytope as manifolds with corners. This completes the proof of Theorem 1.

Acknowledgements The author wishes to thank Professor Mikiya Masuda for his valuable advice about mathematics and his continuing support. Professor Megumi Harada gave the author valuable advice on writing.

\section{References}

[1] D Barnette, Diagrams and Schlegel diagrams, from: "Combinatorial Structures and their Applications", Gordon and Breach, New York (1970) 1-4 MR0270266

[2] V M Buchstaber, T E Panov, Torus actions and their applications in topology and combinatorics, University Lecture Series 24, Amer. Math. Soc. (2002) MR1897064

[3] Y Civan, Some examples in toric geometry, preprint arXiv:math/0306029 
[4] M W Davis, T Januszkiewicz, Convex polytopes, Coxeter orbifolds and torus actions, Duke Math. J. 62 (1991) 417-451 MR1104531

[5] G Ewald, Combinatorial convexity and algebraic geometry, Graduate Texts in Mathematics 168, Springer, New York (1996) MR1418400

[6] H Ishida, Y Fukukawa, M Masuda, Topological toric manifolds, Mosc. Math. J. 13 (2013) 57-98, 189-190 MR3112216

Department of Mathematics, Osaka City University

3-3-138 Sugimoto, Sumiyoshi-ku, Osaka 558-8585, Japan

m13saU0r13@ex.media.osaka-cu.ac.jp

Received: 23 December 2013 Revised: 19 April 2014 